\title{
Editorial
}

\section{MANAGEMENT OF KERATOCONUS}

Alió et al are to be commended for the review entitled 'Keratoconus Management Guidelines', which adds to the previous classic reviews in this field. ${ }^{1-3}$ Keratoconus was described for the first time over 150 years ago by John Nottingham. Over the last few decades, there were significant advances on diagnostic and therapeutic technologies, mostly related to refractive surgery. In addition, the relative high incidence of these disorders have stimulated ectatic diseases be considered as a new subspecialty in ophthalmology. ${ }^{4,5}$

Alió et al are also to be congratulated for having sensitized government leaders to obtain financial support and concern for the prevention and reversal of vision loss associated with ectatic corneal diseases. ${ }^{1}$ Interestingly, this is a trend also seen in other countries, such as France ${ }^{6}$ and Australia. ${ }^{6,7}$ We do advocate for replicating such projects worldwide. In this extensive work, the etiology and epidemiology of keratoconus are reviewed, demonstrating that prevalence and incidence vary among different areas. ${ }^{1}$ This is due to genetic and environmental factors. ${ }^{3,8-10}$ Although considered as a noninflammatory disease, recent histological studies revealed the presence of a sub-acute inflammation process. ${ }^{3,11-13}$ This concept goes in agreement with the current global consensus on keratoconus (GCK), which stated that micro-trauma related to eye rubbing plays a major rule in the pathophysiology of the disease. ${ }^{5}$

Advanced diagnostic technologies have been reviewed, starting from corneal front surface topography. ${ }^{1}$ The advent of three-dimension corneal tomography, providing full pachymetric mapping and posterior surface evaluation has also been considered. ${ }^{14}$ The importance of corneal/anterior segment tomography is also reflected in the GCK, which found agreement from the majority, that abnormalities in the posterior corneal surface and in corneal thickness profile are necessary to diagnose keratoconus. ${ }^{5}$ Biomechanical evaluation holds the promise to be useful for enhancing the accuracy for detecting corneal ectatic diseases. ${ }^{15-18}$ However, the available devices are not yet fully developed. Advances on the interpretation of the generated data or novel technologies, such as the Brillouin microscopy, are also promising. ${ }^{19}$

The need for advanced or enhanced diagnostics is directly related to refractive surgery, so the diagnosis of mild keratoconus is fundamental for screening candidates at high risk for ectasia after laser vision correction. ${ }^{20-22}$ In this section, the authors indirectly address the concept of 'enhanced screening to detect ectasia susceptibility', which was introduced by our study group. ${ }^{21,22}$ The main goal of the enhanced screening of ectasia risk among refractive candidates is the identification of very mild abnormalities, since the referred condition should be likely present in the preoperative state of cases with unexplained ectasia after laser-assisted in situ keratomileusis (LASIK). ${ }^{21,23-25}$ The authors refer to these cases as 'Forme Fruste Keratoconus' (FFKC), which is a good term to describe an abortive form of a disease that may progress or not. ${ }^{21}$ However, FFKC should not be used to describe mild topographic abnormalities, which may be present in a normal patient. ${ }^{26}$ Interestingly, tomography-based algorithms have been demonstrated to augment the diagnostic accuracy of these cases. ${ }^{20,22,27-31}$

The concept of irregular astigmatism, being characterized by ocular wavefront analysis, is well covered. ${ }^{1}$ Wavefront provides additional information related to visual quality and refractive error. We published a study that showed the importance of this technology to facilitate the manifest refraction, determining a significant improvement in visual acuity with sphero-cylindrical lenses in keratoconus patients intolerant to contact lenses. ${ }^{32}$ The potential of combining ocular and corneal wavefront for the diagnosis of milder forms of keratoconus have been recently reported. ${ }^{26}$

Keratoconus classification is also well extensively revised. ${ }^{1}$ The RETICS (Spanish network of research in ophthalmology) classification is described and should be tested in other populations. Interestingly, the GCK also acknowledged the limitations of previously used classifications/staging systems-Amsler-Krumeich and collaborative longitudinal evaluation of keratoconus (CLEK). The current trend is to elaborate studies that correlate clinical findings (i.e. visual performance), with corneal topometric and tomographic parameters are needed. ${ }^{5,33,34}$ Nevertheless, the RETICS classification does not include advanced tomographic data in its methodology. As mentioned previously, visual acuity evaluation in these patients may be challenging due to high levels of irregular astigmatism. Considering a more objective visual function assessment methodology (e.g. assisted by wavefront analysis) would be appropriate for the improvement of this classification. ${ }^{32}$

While the authors review risk factors for keratoconus progression, there is no consensus on the actual definition of ectasia progression. ${ }^{1}$ For example, the GKC agreed that for documenting ectasia progression changes are required in at least two of the following: steepening of the anterior surface, steepening of the posterior surface, thinning or changes in the pachymetric profile. It is worth to note that these changes are a requisite for documenting progression, but the absolute magnitude of the changes is currently unknown and should vary accordingly to the diagnostic tool. ${ }^{5}$ 
The main goals of the treatment of keratoconus patients are: visual rehabilitation and arresting the disease progression. Patient and family education is essential in this topic. The most important nonsurgical treatment measure is verbal guidance regarding the importance of not rubbing the eyes. ${ }^{5,35}$ Treatment of ocular allergy and optimization of ocular surface also plays an essential role. ${ }^{36}$ Visual rehabilitation should be first attempted with spectacles. Contact lenses are the most effective treatment option for visual restoration. This article extensively describes the differences of the various types of contact lenses, which may be used for visual rehabilitation of these patients. ${ }^{1}$ However, this is fundamental to consider that the use of contact lenses must be regarded with caution. ${ }^{5}$ It is worth mentioning that there is no scientific data to support the benefit of contact lenses for decreasing the risk of ectasia progression. ${ }^{37}$

An extensive review of the alternative surgical options, which are less invasive than keratoplasty, is provided. ${ }^{1}$ It is challenging to determine the best surgical approach. ${ }^{35}$ Individual surgical experience, availability of technology and socioeconomic settings influence the clinical decision in different clinical scenarios. ${ }^{5}$ Further interventional and prospective studies are needed to improve the strategies for surgical management, along with elaborating guidelines. Corneal collagen cross-linking (CXL) procedure is described in detail by the authors. ${ }^{1}$ The combination of CXL with other surgical procedures, such as therapeutic customized laser excimer ablation (Athens protocol and Cretan protocol) and intracorneal ring segments (ICRS). ${ }^{35,38,39}$ These combined surgical approaches should be referred as 'Cross-linking plus', once crosslinking is combined with another surgical technique that may allow visual restoration. ${ }^{40}$

Refractive management of keratoconus patients is also well covered. ${ }^{1}$ The differences between therapeutic and elective refractive procedures must be recognized and taken into account. ${ }^{35,41}$ In therapeutic surgery, the measure of success is the improvement in distance-corrected visual acuity, refractive outcome being a secondary goal. In elective refractive surgery, success is measured by uncorrected distance visual acuity, since the goal is reduction or elimination of the need of glasses or contact lenses. Tolerance for refractive correction and anisometropia may determine a therapeutic need in some cases. For example, phakic intraocular lens for myopia correction after ICRS implantation ('Therapeutic Bioptics' concept) is regarded as an elective procedure to reduce the spectacle dependence. ${ }^{35,41,42}$ In these cases, the informed consent process should be performed with appropriate explanation to the patient, who must understand differences between need and possibility, weighing up of the risks, benefits and limitations of the procedure.

The International Journal of Keratoconus and Ectatic Corneal Diseases has devoted a complete issue to publish the review by Alió et al, recognizing it as a definitive review following the ones by Krachmer et al and Rabinowitz. ${ }^{1-3}$ We again commend the authors for the work and suggest this publication as a reference for anyone interested in keratoconus.

\section{REFERENCES}

1. Alio JL, Vega-Estrada A, Peña García P, Durán García ML, Sanz-Díez P. Keratoconus Management Guidelines. Int J Ker Cor Ect Dis 2015;4 (in press).

2. Krachmer JH, Feder RS, Belin MW. Keratoconus and related noninflammatory corneal thinning disorders. Surv Ophthalmol 1984;28(4):293322.

3. Rabinowitz YS. Keratoconus. Surv Ophthalmol 1998;42(4):297-319.

4. Ambrósio RJ. Keratoconus and ectatic corneal diseases: are we facing a new subspeciality? Int J Kerat Ectatic Dis 2012;1(2).

5. Gomes JA, Tan D, Rapuano CJ, Belin MW, Ambrósio R Jr, Guell JL, Malecaze F, Nishida K, Sangwan VS. Group of panelists for the global delphi panel of keratoconus and ectatic diseases. Global consensus on keratoconus and ectatic diseases. Cornea 2015;34(4): 359-369.

6. Asri D, Touboul D, Fournie P, Malet F, Garra C, Gallois A, Malecaze F, Colin J. Corneal collagen crosslinking in progressive keratoconus: multicenter results from the French National Reference Center for Keratoconus. J Cataract Refract Surg 2011;37(12):2137-2143.

7. Kelly TL, Williams KA, Coster DJ. Australian Corneal Graft Registry. Corneal transplantation for keratoconus: a registry study. Arch Ophthalmol 2011;129(6):691-697.

8. Gordon-Shaag A, Millodot M, Shneor E. The epidemiology and etiology of keratoconus. Int J Kerat Ectatic Dis 2012;1(1):7-15.

9. Holland DR, Maeda N, Hannush SB, Riveroll LH, Green MT, Klyce SD, Wilson SE. Unilateral keratoconus: incidence and quantitative topographic analysis. Ophthalmology 1997;104(9):1409-1413.

10. Li X, Rabinowitz YS, Rasheed K, Yang H. Longitudinal study of the normal eyes in unilateral keratoconus patients. Ophthalmology 2004;111:440446.

11. Galvis V, Sherwin T, Tello A, Merayo J, Barrera R, Acera A. Keratoconus: an inflammatory disorder? Eye (Lond) 2015;29(7):843-859.

12. Shetty R, Ghosh A, Lim RR, Subramani M, Mihir K, Reshma AR, Ranganath A, Nagaraj S, Nuijts RM, Beuerman R, et al. Elevated expression of matrix metalloproteinase-9 and inflammatory cytokines in keratoconus patients is inhibited by cyclosporine A. Invest Ophthalmol Vis Sci 2015;56(2):738-750.

13. Cheung IM, McGhee CN, Sherwin T. A new perspective on the pathobiology of keratoconus: interplay of stromal wound healing and reactive species-associated processes. Clin Exp Optom 2013;96(2):188-196.

14. Ambrosio R Jr, Belin MW. Imaging of the cornea: topography vs tomography. J Refract Surg 2010;26(11):847-849.

15. Roberts CJ, Dupps WJ Jr. Biomechanics of corneal ectasia and biomechanical treatments. J Cataract Refract Surg 2014;40(6):991-998 
16. Fontes BM, Ambrosio R Jr, Jardim D, Velarde GC, Nose W. Corneal biomechanical metrics and anterior segment parameters in mild keratoconus. Ophthalmology 2010;117(4):673-679.

17. Luz A FB, Ramos IC, Lopes B, Correia F, Schor P, Ambrósio R. Evaluation of ocular biomechanical indices to distinguish normal from keratoconus eyes. Int J Kerat Ectatic Dis 2012;1(3):145-150.

18. Fontes BM, Ambrosio Junior R, Jardim D, Velarde GC, Nose W. Ability of corneal biomechanical metrics and anterior segment data in the differentiation of keratoconus and healthy corneas. Arq Bras Oftalmol 2010;73(4):333-337.

19. Scarcelli G, Pineda R, Yun SH. Brillouin optical microscopy for corneal biomechanics. Invest Ophthalmol Vis Sci 2012;53(1):185-190.

20. Ambrosio R Jr, Valbon BF, Faria-Correia F, Ramos I, Luz A. Scheimpflug imaging for laser refractive surgery. Curr Opin Ophthalmol 2013;24(4):310-320.

21. Ambrosio R Jr, Randleman JB. Screening for ectasia risk: what are we screening for and how should we screen for it? J Refract Surg 2013;29(4): 230-222.

22. Ambrosio R Jr, Nogueira LP, Caldas DL, Fontes BM, Luz A, Cazal JO, Alves MR, Belin MW. Evaluation of corneal shape and biomechanics before LASIK. Int Ophthalmol Clin 2011;51(2):11-38.

23. Ambrosio R Jr, Jardim D, Netto MV, Wilson SE. Management of unsuccessful LASIK surgery. Compr Ophthalmol Update 2007;8(3): $125-141$.

24. Ambrosio R Jr, Klyce SD, Wilson SE. Corneal topographic and pachymetric screening of keratorefractive patients. J Refract Surg 2003;19(1): 24-29.

25. Ramos IC, Correa R, Guerra FP, Trattler W, Belin MW, Klyce SD, Fontes BM, Schor P, Smolek MK, Dawson DG, et al. Variability of subjective classifications of corneal topography maps from LASIK candidates. J Refract Surg 2013;29(11):770-775.

26. Jafri B, Li X, Yang H, Rabinowitz YS. Higher order wavefront aberrations and topography in early and suspected keratoconus. J Refract Surg 2007;23(8):774-781.

27. Ambrosio R Jr, Caiado AL, Guerra FP, Louzada R, Roy AS, Luz A, Dupps WJ, Belin MW. Novel pachymetric parameters based on corneal tomography for diagnosing keratoconus. J Refract Surg 2011;27(10):753-758.

28. Ambrosio R Jr, Dawson DG, Salomao M, Guerra FP, Caiado AL, Belin MW. Corneal ectasia after LASIK despite low preoperative risk: tomographic and biomechanical findings in the unoperated, stable, fellow eye. J Refract Surg 2010;26(11):906-911.

29. Valbon BF AJR, Glicéria J, Santos R, Luz A, Alves MR. Unilateral Corneal Ectasia after Bilateral LASIK: The Thick Flap Counts. Int J Kerat Ectatic Dis 2013;2(2):79-83.

30. Valbon BDF, Santos RT, Ramos I, Canedo AL, Nogueira L, Ambrosio R Jr. Simplifying ectasia screening with corneal and anterior segment tomography. Rev Bras Oftalmol 2013;72(1):54-58.

31. Glicéria J VB, Valbon BF, Santos RT, Ambrosio Jr R. Pregnancy induced progression of keratoconus in a 37-year-old patient. Int J Kerat Ectatic Dis 2013;2(2):84-88.

32. Ambrosio R Jr, Caldas DL, da Silva RS, Pimentel LN, Valbon BF. Impacto da análise do 'wavefront' na refractometria de pacientes com ceratocone. Rev Bras ophthalmol 2010;69(5):294-300.

33. Lopes BT, Ramos IC, Faria-Correia F, Luz A, Valbon BF, Belin MW, Ambrósio R Jr. Correlation of topometric and tomographic indices with visual acuity in patients with keratoconus. Int J Kerat Ect Cor Dis 2012;1(3):167-172.

34. Salomao MQ, Guerra FP, Ramos IC, Jordao LF, Canedo ALC, Valbon BF, Luz A, Correa R, Lopes B, Ambrósio R Jr. Accuracy of topometric indices for distinguishing between keratoconic and normal corneas. Int J Kerat Ect Cor Dis 2013;2(3):108-112.

35. Faria-Correia F, Luz A, Ambrosio R. Managing corneal ectasia prior to keratoplasty. Exp Rev Ophthalmol 2015;10(1):33-48.

36. Sharma N, Rao K, Maharana PK, Vajpayee RB. Ocular allergy and keratoconus. Ind J Ophthalmol 2013;61(8):407-409.

37. Siqueira JA, Dias LC, Siqueira R, Valbon B, Santos R, Dawson D, Ambrosio R Jr. Long-term improvement after the Athens protocol for advanced keratoconus with significant ectasia progression in the fellow eye. J Kerat Ect Cor Dis 2013;2(3):143-146.

38. Kanellopoulos AJ, Asimellis G. Keratoconus management: long-term stability of topography-guided normalization combined with high-fluence CXL stabilization (the Athens Protocol). J Refract Surg 2014;30(2):88-93.

39. Kymionis GD, Grentzelos MA, Kankariya VP, Pallikaris IG. Combined transepithelial phototherapeutic keratectomy and corneal collagen crosslinking for ectatic disorders: cretan protocol. J Cataract Refract Surg 2013;12(39):1939.

40. Kymionis GD. Corneal collagen cross linking_PLUS. Open Ophthalmol J 2011;5:10.

41. Ambrosio R Jr. Cirurgia refrativa terapêutica: por que diferenciar? Rev Bras ophthalmol 2013;72(2):85-86.

42. Ambrosio R Jr. Intracorneal ring segment, phakic IOL implantation enable keratoconus management without keratoplasty. Ocular Surgery News US Edition 2011. Available at: http://www.healio.com/ophthalmology/cornea-external-disease/news/print/ocular-surgery-news/\%7Be26ba6e3e6db-47e6-8b6a-b53bdcd7f33a\%7D/intracorneal-ring-segment-phakic-iol-implantation-enable-keratoconus-management-without-keratoplasty.

Renato Ambrósio Jr MD PhD

Head, Rio de Janeiro Corneal Tomography and Biomechanics Study Group, Rio de Janeiro Associate Professor, Department of Ophthalmology, Federal University of São Paulo São Paulo, Brazil

Fernando Faria-Correia MD

Research Associate, Rio de Janeiro Corneal Tomography and Biomechanics Study Group Rio de Janeiro, Brazil

Lecturer, Hospital de Braga

PhD Fellow, School of Health Sciences University of Minho, Braga, Portugal 